\title{
Electrotactic Ionic Liquid Droplets
}

By Wayne Francis ${ }^{a}$, Klaudia Wagner ${ }^{b}$, Stephen Beirne ${ }^{b}$, David Officer $^{b}$, Gordon Wallace ${ }^{b}$, Larisa Florea $^{a_{*}}$ and Dermot Diamond ${ }^{a}$

${ }^{a}$ Insight Centre for Data Analytics, National Centre for Sensor Research

Dublin City University,

Dublin 9, Ireland.

${ }^{\mathrm{b}}$ ARC Centre of Excellence for Electromaterials Science and Intelligent Polymer Research Institute, University of Wollongong,

Wollongong, NSW 2522, Australia.

*Corresponding author E-mail: larisa.florea@dcu.ie

\begin{abstract}
To our knowledge, this work describes the first example of electro-guided, selfpropelled droplets composed solely of an ionic liquid (IL), namely trihexyl(tetradecyl)phosphonium chloride $\left(\left[\mathrm{P}_{6,6,6,14}\right][\mathrm{Cl}]\right)$. These self-propelled droplets travel along an aqueous-air boundary to desired destinations within the fluidic network.

Electrotactic movement of the droplets is due to asymmetric electro-stimulated release of a constituent of the IL droplet, the $\left[\mathrm{P}_{6,6,6,14}\right]^{+}$ion, which is a very efficient cationic surfactant, through electrochemically generated $\mathrm{Cl}^{-}$gradients. The direction and speed of movement can be controlled by switching the impressed voltage (typically $5-9 \mathrm{~V}$ ) ON or OFF, and by changing the polarity of the electrodes in contact with the electrolyte solution.

The $\mathrm{Cl}^{-}$gradients required for droplet movement are electrochemically generated using 3D printed electrodes which are embedded within the fluidic channels. On demand creation of these $\mathrm{Cl}^{-}$gradients electrochemically allows reversible droplet movement over expended periods of time, and provides a means for precise control over the droplet trajectory.
\end{abstract}

Keywords: (Ionic Liquids, Electrotaxis, Droplets, Surface Tension) 


\section{Introduction}

The movement of cells and other biological entities is essential to the survival of all life. This is true from single celled organisms to the largest and most complex forms of life such as mammals. Fluid and cells are transported throughout large complex bodies through active pumping, while nutrients are transported in and out of cells through the use of special biological "microvehicles" known as vesicles. Inside the cell there are motor proteins which shuttle cargo around the cytosol, such as specialised walking molecules known as kinesins [1]. Helicase is another unique biological motor whose main function is to separate two annealed nucleic acid strands (for example within DNA or RNA) [2]. This type of micromolecular movement is triggered and controlled through chemical and electrical impulses.

Many single cell and multicellular organisms move in response to chemical stimuli present in their environment [3], a phenomenon known as chemotaxis. Prokaryotic cells such as bacteria use chemotaxis to find sources of food or flee harmful toxins, while eukaryotic and somatic cells have much more complex functions which rely on this behaviour. For example, lymphocytes use chemotaxis behaviour to follow a chemical trail left by a pathogen and then destroy the harmful intruder $[4,5]$. The passage of vesicles carrying cargo in and out of the cell relies on chemical gradients to transfer their cargo [6], while other examples of chemically driven biological motors include enzymes that can follow a chemical signal to the corresponding substrate [7], and sperm cells, which use prominent flagella composed of a core of microtubules, powered by flagellar dynein, to propel themselves through the female reproductive tract [8].

The movement of biological organisms is not solely triggered through chemical stimuli; in fact, much of the movement of animal and plant cells is due to the generation of an electric current. For example, in mammals, the onset of wound healing is due to weak electric currents which are generated immediately upon receiving the wound [9]. Humans and 
mammals are found to have a natural electrical gradient across healthy, intact skin due to the secretion of chloride $\left(\mathrm{Cl}^{-}\right)$ions and the absorption of sodium $\left(\mathrm{Na}^{+}\right)$ions, which results in the outer layers of the skin being more negatively charged than the inner layers. Upon receiving a wound (sufficient to cause skin breakage) there is a flow of positively charged ions emitted from the wound that generates an electric current perpendicular to the skin at the site of the wound [9]. This electric current induces the migration of keratinocytes and mammalian epithelial cells to the wound and triggers the onset of healing [10]. Movement of cells in response to an electric field is termed galvanotaxis or electrotaxis [11]. Synthetically induced electrotaxis is mainly used to direct the movement of cells in both in-vitro and in-vivo experiments, as it offers better control over on-demand movement of cells compared to solely controlling movement through chemotaxis [12]. An exciting use of electrotactic-guided cells is in-vivo wound healing, in which cells are guided to specific destinations to encourage the growth or repair of damaged tissue [9]. Other groups have made impressive progress towards using electro-guided cells for the repair of the brain and central nervous system $[13,14]$. Treatment of neurological diseases with electrical stimulation has advanced significantly in recent years, for example through demonstrations that electric fields can enhance neuroplasticity processes. When electric fields within the physiological range were applied in-vitro the movement of several different neural cells types was induced. This ability to induce the movement of specific neural cells to specific destinations with the added benefit of enhancing neuroplasticity processes could potentially give rise to dramatic improvements in the treatment of neurological and psychiatric diseases [13].

Although the chemotactic and electrotactic response of living organisms has been extensively researched, there is a notable lack of reports into synthetic approaches that mimic these forms of movement. Inspired by the electrotactic movement of living organisms, here 
we describe for the first time, simple synthetic droplets composed solely of an ionic liquid that can be guided at the liquid-air interface using electro-generated ion gradients.

In recent years, multiple approaches have been investigated for the generation of micro-droplet movement, including alteration of the wettability of the substrate through chemical $[15,16]$ or electrochemical means $[17,18]$. Other forms of stimuli, including temperature [19], magnetic [20], light [21], and acoustic forces [22] have also been used for the actuation and guidance of micro-droplets. Although these approaches permit individual droplet control through external stimulation, they generally require relatively complicated experimental arrangements [17] or complex droplet compositions [23].

Surfactant release has been employed as another contactless method for controlled movement of droplets that rest at the liquid/air interface [23]. When surfactants are released into an aqueous system, they locate at the liquid/air interface with the polar heads embedded in the water phase and the non-polar tails projecting out of the solution. The release of surfactant lowers the surface tension of the system, and once this occurs, a spontaneous movement of liquid is created from areas of low surface tension, to areas of high surface tension, a phenomenon known as the Marangoni effect [24]. By creating conditions wherein asymmetrical release from the droplet of a pre-loaded surfactant occurs, directional control over the droplet movement can be achieved. Using this premise, "smart droplets" were developed which can solve complex mazes by following a pre-established $\mathrm{pH}$ gradient [23]. Photo-controlled droplet movement was also achieved by asymmetric release of surfactant through localised creation of a pH gradient using a photo-acid generator [25]. Another approach involved the use of photo-responsive azobenzene surfactants for droplet manipulation at the air/liquid interface [26]. Electrochemical control over the surface tension of a liquid metal was also demonstrated through the formation and removal of a surface oxide on liquid metals by applying low voltages $(<1 \mathrm{~V})$ [27]. When the surface oxide is present on 
the liquid metal it acts as a surfactant and significantly lowers the interfacial tension between the liquid metal and the electrolyte solution (from $\sim 500 \mathrm{~mJ} / \mathrm{m}^{2}$ to near zero). Removal of the surface oxide restores the surface tension of the liquid metal. This technique allowed for shape-reconfiguration of liquid metals alloys of gallium and their guidance through a fluidic channel.

Previously we reported chemotactic droplets composed solely of an ionic liquid namely trihexyl(tetradecyl)phosphonium chloride $\left(\left[\mathrm{P}_{6,6,6,14}\right][\mathrm{Cl}]\right)$ that moved due to triggered release of the cationic surfactant $\left[\mathrm{P}_{6,6,6,14}\right]^{+}$and were guided to specific destinations through chemically generated ion gradients [28]. Ionic liquids are ideal solvents for the development of smart vessels and "vehicles" due to their negligible vapour pressure, high thermal stability and low combustibility, making them significantly more stable when compared to conventional droplet systems based on organic solvents. [29] $\left[\mathrm{P}_{6,6,6,14}\right][\mathrm{Cl}]$ in particular has a high viscosity and is relatively hydrophobic which means that it can form stable droplets on aqueous/air interfaces [29]. Ionic liquids have also been previously shown to exhibit extended electrochemical windows [30-33]. Buzzeo et al. [33] examined the electrochemical window of several Ionic liquids (1-ethyl-3-methylimidazolium bis(trifluoromethylsulfonyl)imide $\left(\left[\mathrm{C}_{2} \operatorname{mim}\right]\left[\mathrm{NTf}_{2}\right]\right), n$-hexyltriethylammonium bis(trifluoromethylsulfonyl)-imide $\left(\left[\mathrm{N}_{6,2,2,2}\right]\left[\mathrm{NTf}_{2}\right]\right)$, 1-hexyl-3-methylimidazolium trifluorotris-(pentafluoroethyl)phosphate $\left(\left[\mathrm{C}_{6} \mathrm{mim}\right][\mathrm{FAP}]\right)$ and tris $(n$-hexyl0tetradecylphosphonium trifluorotris(pentafluoroethyl)phosphate $\left.\left(\left[\mathrm{P}_{6,6,6,14}\right][\mathrm{FAP}]\right)\right)$ using cyclic voltammetry at gold and platinum microelectrodes (diameter of $10 \mu \mathrm{m}$ ). When the neat ILs were used as electrolytes, $\left[\mathrm{P}_{6,6,6,14}\right][\mathrm{FAP}]$ showed no redox activity in the range of $-5 \mathrm{~V}$ to $5 \mathrm{~V}$ at the gold electrode, and no redox activity in the range of -4 to $4 \mathrm{~V}$ at the platinum electrode. The electrochemical window of this IL could potentially extend over a wider range, however these were the limits set for the experiments performed by Buzzeo et al.. No redox activity was observed for 
$\left[\mathrm{N}_{6,2,2,2}\right]\left[\mathrm{NTf}_{2}\right]$ between $-4 \mathrm{~V}$ and $4 \mathrm{~V}$ for both electrodes and no activity was shown for both $\left[\mathrm{C}_{2} \mathrm{mim}\right]\left[\mathrm{NTf}_{2}\right]$ and $\left[\mathrm{C}_{6}\right.$ mim $][\mathrm{FAP}]$ between $-2 \mathrm{~V}$ and $2 \mathrm{~V}$ (at both electrodes). This extended electrochemical window shows that ILs are stable electrolytes for use across a broad potential range.

Herein we report reversible electrotactic movement of the same $\left[\mathrm{P}_{6,6,6,14}\right][\mathrm{Cl}]$ droplets to specific destinations through electrochemically generated $\mathrm{Cl}^{-}$gradients. To our knowledge this is the first demonstration of spontaneous electrotactic movement of droplets composed solely of an IL at the aqueous/liquid interface. The electrotactic movement presented is not only reversible and repeatable, but the droplet trajectory and speed can be controlled by electrode polarity and applied potential, respectively.

\section{Materials and Methods}

\subsection{Materials}

Sodium chloride $(\mathrm{NaCl})$ (Sigma-Aldrich ${ }^{\circledR}$ Ireland Ltd), trihexyl(tetradecyl)phosphonium chloride $\left(\left[\mathrm{P}_{6,6,6,14}\right][\mathrm{Cl}]\right)$ (Sigma-Aldrich ${ }^{\circledR}$ Ireland Ltd), $N$, $N$-Dimethyl-9,9'-biacridinium dinitrate (lucigenin) (Sigma-Aldrich ${ }^{\circledR}$ Ireland Ltd) and 1(methylamino)anthraquinone red dye (Sigma-Aldrich® Ireland Ltd) were all used as purchased.

\subsection{Micro-channel and electrodes fabrication}

The titanium mesh electrodes $(4 \times 4$ electrodes, each with a diameter of $3.5 \mathrm{~mm}$ and 8 $\mathrm{mm}$ in height) were 3D printed using a Realizer SLM-50 printer (Figure 1). 3D printing produces high surface area electrodes while also allowing facile integration of the electrodes 
into a single platform, as the design and shape of the individual electrodes can be tuned to match the design of the fluidic channel.

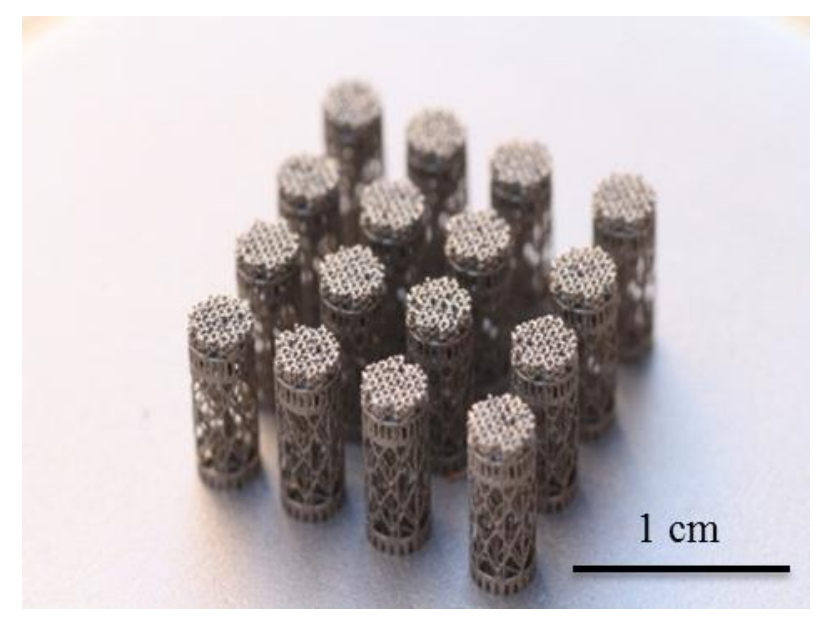

Figure 1. 3D printed titanium mesh electrodes.

Following printing, the electrodes were embedded into fluidic channels. Two different types of channels were used. In the first case, the channels were designed in Solid Works CAD modelling software and fabricated in acrylate ABS-Like ${ }^{\mathrm{TM}}$ Polyjet photopolymer (Stratasys) using an Objet350 Connex 3D printer. The channels were $2.5 \mathrm{~mm}$ deep, $3 \mathrm{~mm}$ in width and the distance between opposing electrodes was $16 \mathrm{~mm}$, see Figure 2a. A narrow channel which was $0.5 \mathrm{~mm}$ wide and $1 \mathrm{~mm}$ in length was placed between the electrodes and the fluidic system in order to prevent the droplet from crushing into the electrodes.

In the second case, the channels were designed in AutoCAD 2014, and a $\mathrm{CO}_{2}$ laser ablation system (Epilog Zing Laser Series) was used to cut the channels in $1 \mathrm{~mm}$ poly(methylmethacrylate) (PMMA) sheet that had a $50 \mu \mathrm{m}$ double-sided pressure-sensitive adhesive (PSA) layer pre-attached. Once cut, the protective layer from the PSA was removed and the PMMA/PSA layer was laminated with another $1 \mathrm{~mm}$ PMMA sheet. The obtained channels were $2 \mathrm{~mm}$ wide, $1 \mathrm{~mm}$ high, with a $2 \mathrm{~cm}$ distance between the electrodes and a 4 mm diameter hole for electrode placement, see Figure $\mathbf{2 b}$.

For both types of channels, following printing, the 3D mesh titanium electrodes were inserted into the placement holes incorporated into the channel design. The electrodes were 
then secured in position using UV curable glue and the channel was placed onto a breadboard to facilitate the connection of the extruding pins from electrodes to the wires connecting to the power supply. Two different channel configurations for electro-stimulation of droplets were obtained (Figure 2).
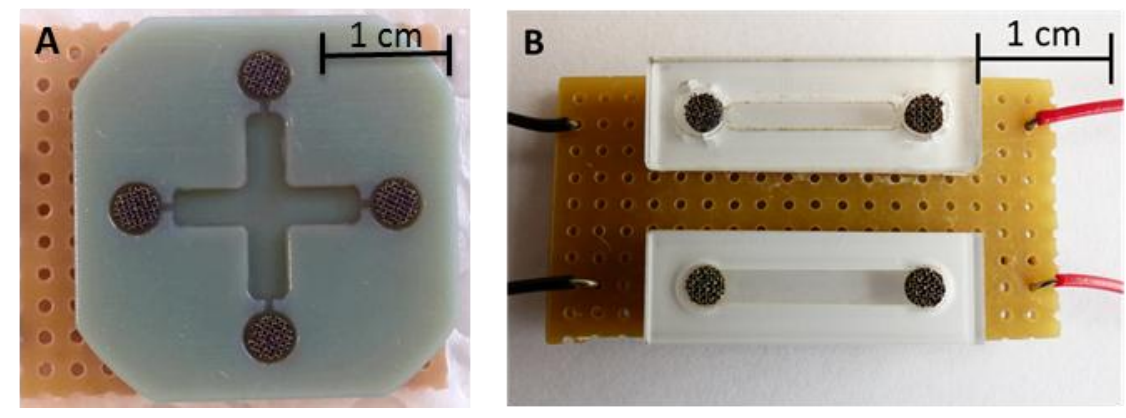

Figure 2. Channels for electro-stimulation of droplets showing embedded 3D printed titanium electrodes; A) Cross-shape 3D printed channels in ABS Polyjet Photopolymer (Stratasys) based on acrylic monomers B) Laser cut PMMA channel.

\subsection{Droplet movement}

To achieve electrotactic droplet movement, the channels were initially filled with electrolyte solution of varying concentration, $10^{-2} \mathrm{M} \mathrm{NaCl}$ and $10^{-3} \mathrm{M} \mathrm{NaCl}$, respectively. The voltage was then applied to the electrodes $(5-9 \mathrm{~V})$; after $10-30 \mathrm{~s}$ the droplet was placed at the cathode (-) and spontaneously moved towards the anode (+). When the polarity of the active electrodes was reversed, the droplets moved in the opposite direction.

\subsection{Current generation study}

In order to study the current generated when applying a voltage across the $\mathrm{NaCl}$ solution, the channels were initially filled with electrolyte solution $\left(10^{-2} \mathrm{M} \mathrm{NaCl}\right.$ and $10^{-3} \mathrm{M}$ $\mathrm{NaCl}$, respectively) and the required voltage was supplied to the electrodes $(1-10 \mathrm{~V})$. The current range was then measured using an M Digital Multimeter (N73CG). 


\section{5 $\mathrm{Cl}^{-}$concentration gradient visualisation}

In order to visualise the generation of the $\mathrm{Cl}^{-}$gradient, a $10^{-3} \mathrm{M} \mathrm{NaCl}$ solution containing $10^{-4} \mathrm{M}$ lucigenin was made and placed in the straight PMMA fluidic channel (the channels were $2 \mathrm{~mm}$ wide, $1 \mathrm{~mm}$ high, with a $2 \mathrm{~cm}$ distance between the electrodes) and 9V was applied across the solution. To demonstrate the reversibility of the $\mathrm{Cl}^{-}$gradient, the polarity of the electrodes was switched after the desired time. A $369 \mathrm{~nm}$ LED was used to excite the fluorescent lucigenin dye. Videos of the gradient generation were captured using a Panasonic DMC-FZ200 camera.

\section{Results and Discussion}

\subsection{Droplet movement}

The droplets used in this study were composed solely of the ionic liquid $\left[\mathrm{P}_{6,6,6,14}\right][\mathrm{Cl}]$ and a small amount of red dye (1-(methylamino)anthraquinone) used only for better visualisation. Due to the strong association of the $\mathrm{Cl}^{-}$anion with the $\left[\mathrm{P}_{6,6,6,14}\right]^{+}$cation, the IL is highly hydrophobic and is able to form a stable droplet at the air-water interface [34]. The movement of the droplet is due to the controlled asymmetric release of the sparingly soluble $\left[\mathrm{P}_{6,6,6,14}\right]^{+}$, which behaves as a cationic surfactant at the air-water boundary. Previously we have shown that when $1 \mathrm{mg} / \mathrm{ml}$ of this IL was placed into a $10^{-2} \mathrm{M}$ solution of $\mathrm{NaCl}$ only a negligible drop in surface tension was observed (from $\sim 73.9$ to $\sim 73.2 \mathrm{mN} / \mathrm{m}$ ). However, when the same volume of the IL was placed into a lower concentration of this electrolyte $(e . g$. $10^{-5} \mathrm{M} \mathrm{NaCl}$ ) there was a large drop in surface tension (from $\sim 74.2$ to $\sim 38.4 \mathrm{mN} / \mathrm{m}$ ) [28]. This shows that the $\left[\mathrm{P}_{6,6,6,14}\right]^{+}$does behave as a strong cationic surfactant preferentially in low ionic strength solutions. When $\left[\mathrm{P}_{6,6,6,14}\right]^{+}$diffuses from the droplet into the aqueous solution, its surfactant character causes a sudden drop of the surface tension of the solution [28]. The rate of release of surfactant is controlled by the solubility of the $\mathrm{Cl}^{-}$ion, as any $\mathrm{Cl}^{-}$ 
transfer from the droplet to the aqueous phase must be balanced by an equivalent transfer of $\left[\mathrm{P}_{6,6,6,14}\right]^{+}$to maintain overall charge neutrality. Release of $\mathrm{Cl}^{-}$ions from the droplet depends on the local aqueous $\mathrm{Cl}^{-}$concentration at the IL-aqueous phase boundary. Hence, in the presence of an aqueous phase $\mathrm{Cl}^{-}$gradient, differential release of $\left[\mathrm{P}_{6,6,6,14}\right]^{+}$occurs across the droplet boundary and a surface tension gradient is created which generates Marangoni-like liquid flow causing the droplet to move towards areas of higher surface tension (i.e. lower $\left.\left[\mathrm{Cl}^{-}\right]\right)$.

In this study, the $\mathrm{Cl}^{-}$gradients within the electrolyte solutions were electrochemically generated. When an external electric field is applied across a $\mathrm{NaCl}$ solution, a $\mathrm{Cl}^{-}$ concentration gradient is created as the $\mathrm{Na}^{+}$ions migrate towards the cathode and $\mathrm{Cl}^{-}$ions towards the anode. When a $\left[\mathrm{P}_{6,6,6,14}\right][\mathrm{Cl}]$ droplet is placed on the surface of this solution, the $\mathrm{Cl}^{-}$concentration gradient causes an asymmetrical release of the $\left[\mathrm{P}_{6,6,6,14}\right]^{+}$cationic surfactant which propels the droplet towards the anode. Furthermore, the applied electric field may cause a Faradic rearrangement of the charged ions within the IL droplet (Figure 3), thereby creating ion concentration gradients which may reinforce the droplet movement mechanism [35]. This phenomenon occurs due to the bipolar electrode (BPE) effect, through which Faradic reactions can occur at both ends of the BPE in the presence of a sufficiently high electric field, creating localized net charges (poles), even though the BPE is unconnected to an external power source [35]. However, due to the very strong ion-pairing within the IL [34], the contribution of this effect to the droplet movement is likely to be much smaller than that of the ion gradient in the aqueous phase.

Hence, when a voltage is applied $(5-9 \mathrm{~V})$, a $\left[\mathrm{P}_{6,6,6,14}\right][\mathrm{Cl}]$ droplet positioned near to a cathode (-) will release the cationic surfactant $\left[\mathrm{P}_{6,6,6,14}\right]^{+}$in an asymmetrical manner, creating Marangoni like flows which drive the droplet towards the anode (+) (Figure 3). 


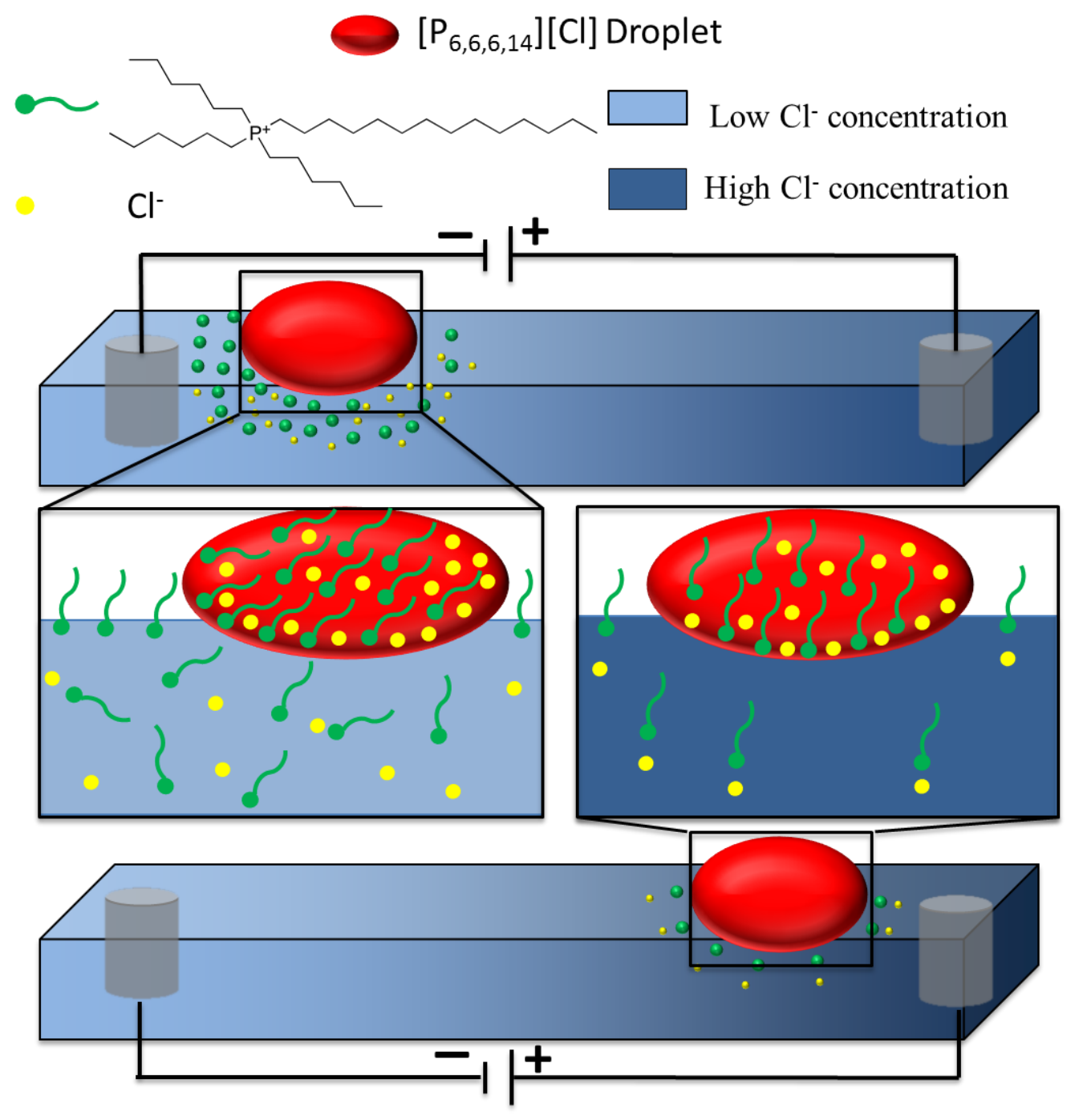

Figure 3. Relative solubility of the surfactant $\left[P_{6,6,6,14}\right][\mathrm{Cl}]$ droplet in lower $\mathrm{Cl}$ concentration (at the cathode, top) and in higher $\mathrm{Cl}^{\circ}$ concentration (at the anode, bottom).

Using this approach we have shown that the droplets can be very effectively guided along channels from the cathode (-) towards the anode (+). In addition to reversing direction within a channel, the droplets can be 'steered' into side channels at junctions, by polarizing appropriate electrode pairs. This can be seen in Figure 4, which shows a droplet being guided to each electrode twice within the fluidic network (See Video S1, Supplementary data). 


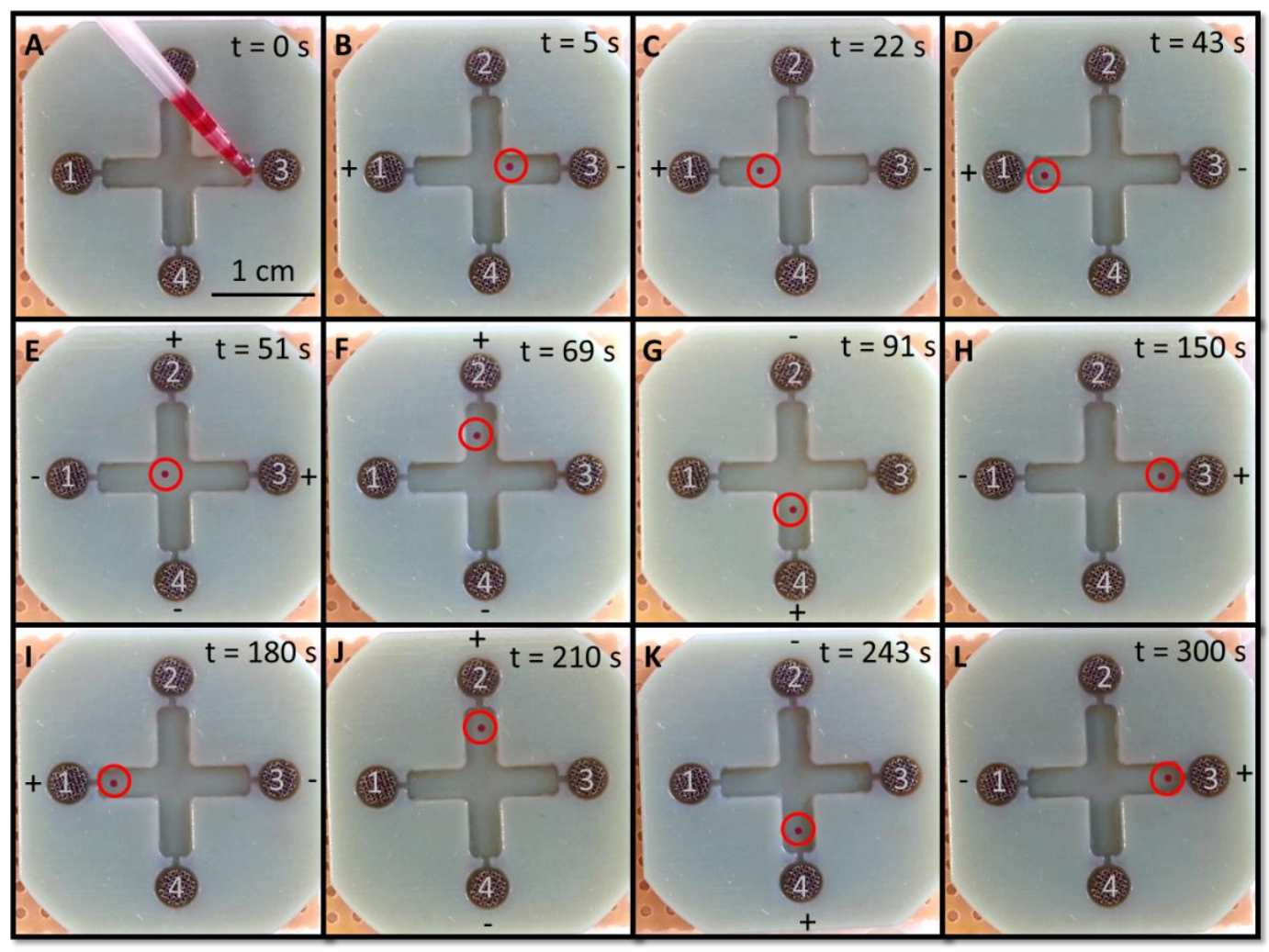

Figure 4. Sequence of video frames demonstrating controlled movement of the $\left[P_{6,6,6,14}\right][\mathrm{Cl}]$ droplet using potential differences applied across pairs of electrodes 1-4. The channels are filled with a solution of $10^{-3} \mathrm{M} \mathrm{NaCl}$ and a $9 \mathrm{~V}$ potential difference is applied across selected electrode pairs. AIntroduction of the droplet onto the aqueous solution; $B-C$ - The droplet is propelled from the initial cathode (3) towards the opposite anode (1); D - The droplet arrives at electrode (1); E - The polarity of the electrodes is then reversed and the droplet moves back towards electrode (3); $F-A s$ the droplet approaches the centre point, the, potential difference is applied across electrodes (2 cathode) and (4 - anode) and the droplet is diverted into the cross channel; $G$-Upon arrival at electrode (2) the polarity is reversed, and the droplet moves towards electrode (4); $H$-Using a similar approach, the droplet is made to return to the original starting position. I-L The process is repeated, showing that the droplet can be moved to each electrode multiple times. (For full video see ESI, video S1)

\subsection{Current generation study}

Figure 5 show the current values obtained when $1-10 \mathrm{~V}$ was applied between neighbouring electrodes (distance between electrodes $=2 \mathrm{~cm}$ ) for different concentrations of $\mathrm{NaCl}\left(10^{-2} \mathrm{M}\right.$ and $10^{-3} \mathrm{M}$, respectively). Despite the difference in current observed for each concentration, unidirectional movement of the droplet was not achieved unless a voltage of 5 $\mathrm{V}$ or higher was applied. It was also noted that for $10^{-2} \mathrm{M} \mathrm{NaCl}$ solution, bubbles due to electrolysis were visible at the cathode when the applied voltage was $7 \mathrm{~V}$ or greater. In the case of the $10^{-3} \mathrm{M} \mathrm{NaCl}$ solution, this was not observed for voltages below $10 \mathrm{~V}$. Bubble 
generation due to electrolysis disrupts droplet movement in the vicinity of the electrodes, and therefore electrotactic droplet movement experiments were performed and analysed below these threshold values. No electrolysis of the electrolyte solution is observed under our experimental conditions because of a combination of two parameters; the first is the pacification of the titanium electrodes (with titanium oxide) which greatly reduces the efficiency of the electrode and secondly is the low current density of the electrolyte solutions which also impedes electrolysis.

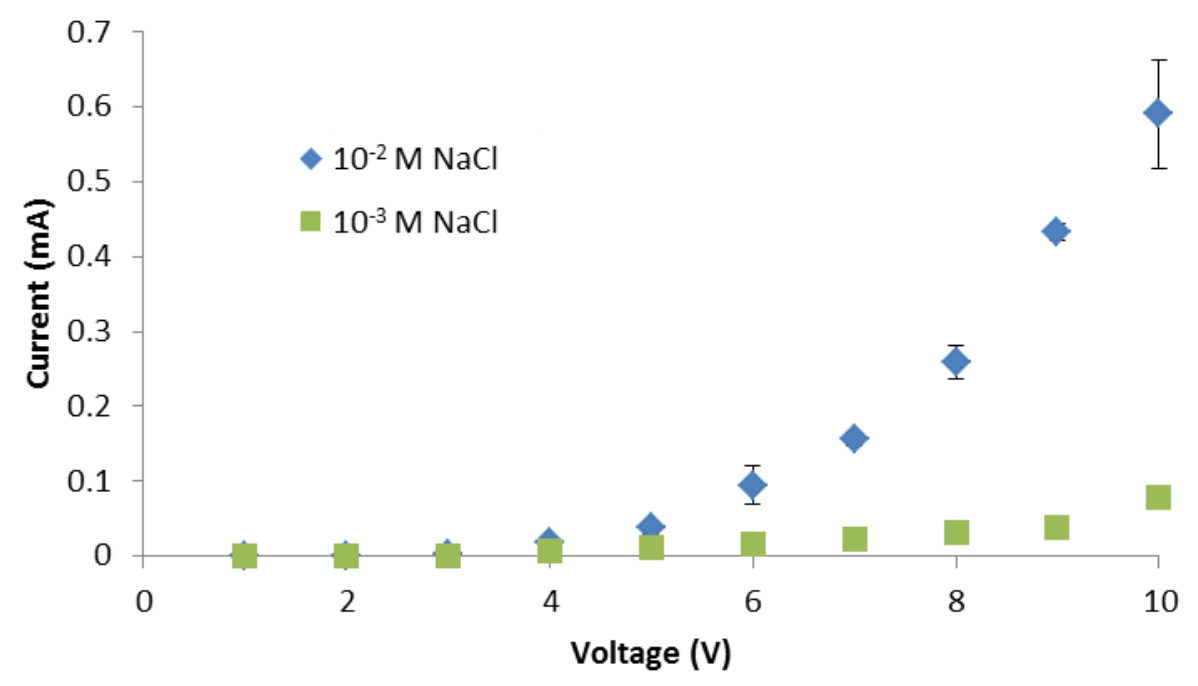

Figure 5. Current generated when the applied voltage was varied over the range $1-10 \mathrm{~V}$ for $10^{-2} \mathrm{M}$ and $10^{-3} \mathrm{M} \mathrm{NaCl}$ solutions, respectively. Error bars are standard deviations for $n=3$ replicate measurements. Unidirectional movement was first observed at $5 \mathrm{~V}$ for all of the solutions. Visible gas generation was first observed at the electrodes at $7 \mathrm{~V}$ for $10^{-2} \mathrm{M}$ solutions and at $10 \mathrm{~V}$ for the $10^{-3} \mathrm{M}$ solutions.

As expected, the current increased as the voltage was raised above $c a .5 \mathrm{~V}$, and the current obtained increased significantly with electrolyte concentration. The speed of the droplet movement was also dependent on a number of parameters, which include the time at which the droplet was placed (in relation to the moment the potential was applied), the volume of the droplet and the interfacial contact area between the droplet and the aqueous solution. However, when these parameters were held relatively constant, an increase in droplet speed was observed with increasing applied voltage (below visible gas generation values). For 
example, droplet speeds of $1-2 \mathrm{~mm} \mathrm{~s}^{-1}$ were obtained when a potential of $9 \mathrm{~V}$ was applied to a $10^{-3} \mathrm{M} \mathrm{NaCl}$ solution. No controlled movement of the droplet was observed at any of the $\mathrm{NaCl}$ electrolyte concentrations studied until voltages of at least $5 \mathrm{~V}$ were applied. These results indicate that unless an ion gradient is generated across the length of the channel (by means of the applied voltage), no asymmetrical release of surfactant from the droplet into the aqueous solutions occurs, and no droplet movement happens. Rather, in the absence of the ion gradient, either no movement or random motion of the droplet is observed.

\section{3 $\mathrm{Cl}^{-}$concentration gradient visualisation}

In order to confirm the presence of a $\mathrm{Cl}^{-}$gradient within the channel, a study was performed using the florescent dye lucigenin. The fluorescence of lucigenin is highly quenched by $\mathrm{Cl}^{-}$, and therefore if a $\mathrm{Cl}^{-}$gradient is created in the channel via the applied voltage, a gradient in the fluorescence of the solution should also be observed. The PMMA channels were filled with a solution of $10^{-3} \mathrm{M} \mathrm{NaCl}$ containing $10^{-4} \mathrm{M}$ lucigenin and a 369 nm led light source was used to excite the dye. The experiment was recorded in a dark room. As can be seen in Figure 6 and video S2 (Supplementary data), approximately 10 minutes after application of $9 \mathrm{~V}$ across the electrodes, a fluorescent gradient becomes visible in the channel. This continues to develop, and is fully formed and stable after approximately 30 minutes. Video S3 (Supplementary data) shows that the formation of the fluorescent gradient is reversible. In this case, after allowing $\mathrm{Cl}^{-}$gradient formation for $10 \mathrm{~min}$ at an applied voltage of $9 \mathrm{~V}$, the polarity of the electrodes was reversed. It was observed that after additional 10 minutes the fluorescent gradient also had reversed. Video S4 (Supplementary data) shows the same experiment performed with a solution of $10^{-4} \mathrm{M}$ lucigenin in deionised water. The video shows that despite the $9 \mathrm{~V}$ applied voltage across the solution, there was no visible difference in the fluorescence across the channel due to the absence of $\mathrm{Cl}^{-}$ions in 
solution. The visualisation of a fluorescent gradient in the $\mathrm{NaCl}$ solutions and the absence of one in water is further proof for the formation of an extended $\mathrm{Cl}^{-}$gradient in the fluidic channel. This extended gradient has also been shown previously when $\mathrm{NaCl}$ was used as the electrolyte for electro-controlled actuation of polyelectrolyte-doped gels that were submerged in an ionic solution. Application of an electrical potential $\left(5 \mathrm{~V} \mathrm{~cm}^{-1}\right)$ between two graphite electrodes placed $6 \mathrm{~cm}$ apart created an asymmetrical redistribution of the mobile ions causing osmotic pressure differences inside the gel and therefore asymmetric swelling inducing unidirectional movement of the hydrogel "walker" [36].

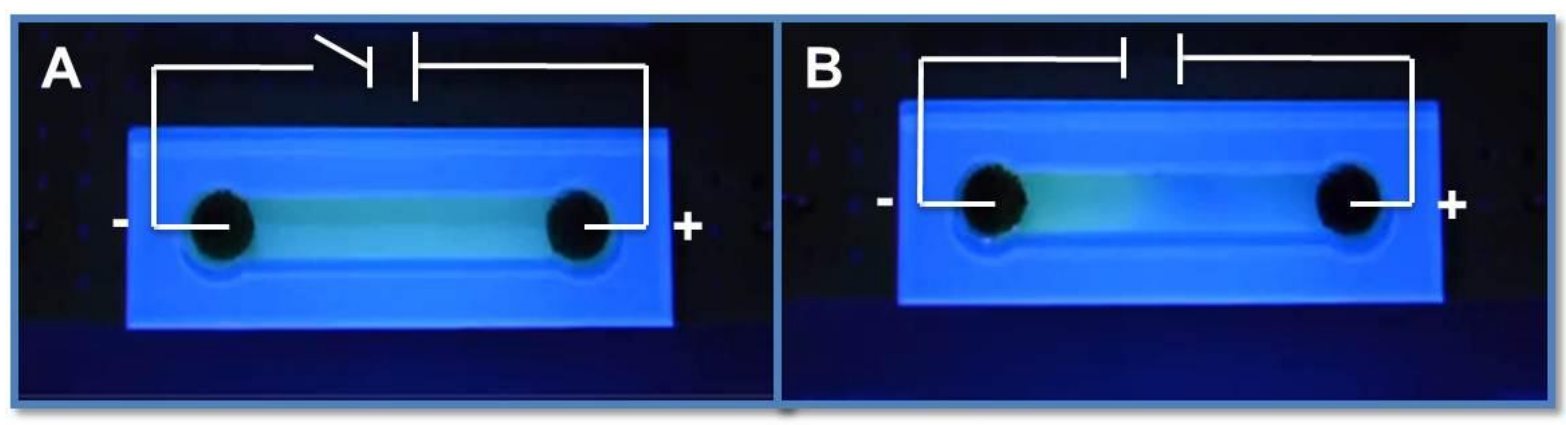

Figure 6: Snapshots showing the fluorescent gradient due to quenching of the lucigenin dye by $\mathrm{Cl}^{-}$ anions in the case when a solution of $10^{-3} \mathrm{M} \mathrm{NaCl}$ containing $10^{-4} \mathrm{M}$ lucigenin was used. A) The fluorescence of the solution when no voltage is applied. B) The fluorescence of the solution when $9 \mathrm{~V}$ was applied for 30 minutes.

This visualisation study supports the contention that the movement of the droplets is due to asymmetrical release of $\left[\mathrm{P}_{6,6,6,14}\right]^{+}$cationic surfactant, which is due to the presence of an electrochemically generated $\mathrm{Cl}^{-}$gradient. Control studies were also performed in which a similar IL, trihexyl(tetradecyl)phosphonium dicyanamide $\left(\left[\mathrm{P}_{6,6,6,14}\right][\mathrm{DCA}]\right)$, was investigated under the same conditions as the $\left[\mathrm{P}_{6,6,6,14}\right][\mathrm{Cl}]$ droplet. No significant movement of the $\left[\mathrm{P}_{6,6,6,14}\right][\mathrm{DCA}]$ was observed as the $\mathrm{DCA}^{-}$anion is much less soluble in the aqueous solution compared to the $\mathrm{Cl}^{-}$anion [29], and release of the surfactant $\left[\mathrm{P}_{6,6,6,14}\right]^{+}$from the droplet is correspondingly supressed. 
ILs have been shown to be excellent solvents $[37,38]$ and are able to solubilise and therefore transport many organic [39] and inorganic materials, and biomaterials such as enzymes, proteins, amino acids, peptides. This further broadens the potential for these electrotactic droplets to perform advanced functions such as being used as micro-vessels for chemical reactions at pre-determined locations, dynamic sensing units, cargo carriers and possible drug-delivery systems. These properties make them better suited as micro-scale "vehicles" compared to conventional organic solvent droplets, which have a limited life-time due to evaporation. Traditional organic droplets also require further addition of surfactant to achieve movement, while the movement of the IL droplets is determined entirely by the inherent surfactant behaviour of the IL itself.

\section{Conclusion}

In conclusion, we have demonstrated that simple single component IL droplets can move spontaneously and be guided to multiple destinations within a fluidic chip in the presence of electrochemically generated $\mathrm{Cl}^{-}$gradients. Electrotactic droplets allow for reversible movement and permit the user to change the course of the droplet by simply changing which electrodes are being addressed within the chip. Movement of electrotactic droplets require relatively simple means of achieving actuation i.e by simply inserting a small number of electrodes within a fluidic chip $(2-4)$ and by applying relatively low voltages $(5-$ $9 \mathrm{~V})$. Since there is already a large and ever growing library of ILs available, this opens up the possibility of creating electrotactic droplets with "designer" properties [29]. Some ILs offer attractive poosibilities for electrochemical experiments, via extended electrochemical windows in aqueous media [30-33] and stability across a broad voltage range. Furthermore, numerous droplet functions can be realised based on IL abilities to carry a wide range of solutes (reaction precursors, drug components, etc.), including spatial and temporal control of 
reactions within fluidics systems by merging droplets at pre-determined locations or through

triggered release of cargo at specific locations.

\section{Acknowledgements}

This work has been supported by Science Foundation Ireland under Insight initiative, grant SFI/12/RC/2289 and European Union Marie Curie People Programme Mask: PIRSES-GA2010-269302, and the Australian Research Council Centre of Excellence Scheme (Project Number CE 140100012). The authors also wish to acknowledge the Australian National Fabrication Facility (ANFF) for access to design and additive fabrication capabilities. The authours would like to acknowledge the helpful input from Prof. Robert Forster to the manuscript.

\section{References}

[1] L.S. Goldstein, Z. Yang, Microtubule-based transport systems in neurons: the roles of kinesins and dyneins, Annu Rev Neurosci, 23(2000) 39-71.

[2] M. Yoneyama, M. Kikuchi, T. Natsukawa, N. Shinobu, T. Imaizumi, M. Miyagishi, et al., The RNA helicase RIG-I has an essential function in double-stranded RNA-induced innate antiviral responses, Nat Immunol, 5(2004) 730-7.

[3] P.J. Van Haastert, P.N. Devreotes, Chemotaxis: signalling the way forward, Nature reviews Molecular cell biology, 5(2004) 626-34.

[4] C.G. Larsen, A.O. Anderson, E. Appella, J.J. Oppenheim, K. Matsushima, The neutrophil-activating protein (NAP-1) is also chemotactic for T lymphocytes, Science, 243(1989) 1464-6.

[5] J.Y. Wu, L. Feng, H.-T. Park, N. Havlioglu, L. Wen, H. Tang, et al., The neuronal repellent Slit inhibits leukocyte chemotaxis induced by chemotactic factors, Nature, 410(2001) 948-52.

[6] P. Naccache, H. Showell, E. Becker, R. Sha'afi, Transport of sodium, potassium, and calcium across rabbit polymorphonuclear leukocyte membranes. Effect of chemotactic factor, The Journal of cell biology, 73(1977) 428-44.

[7] S. Funamoto, R. Meili, S. Lee, L. Parry, R.A. Firtel, Spatial and temporal regulation of 3phosphoinositides by PI 3-kinase and PTEN mediates chemotaxis, Cell, 109(2002) 611-23.

[8] M. Spehr, G. Gisselmann, A. Poplawski, J.A. Riffell, C.H. Wetzel, R.K. Zimmer, et al., Identification of a testicular odorant receptor mediating human sperm chemotaxis, Science, 299(2003) 2054-8.

[9] M. Zhao, B. Song, J. Pu, T. Wada, B. Reid, G. Tai, et al., Electrical signals control wound healing through phosphatidylinositol-3-OH kinase- $\gamma$ and PTEN, Nature, 442(2006) 457-60.

[10] R. Nuccitelli, A role for endogenous electric fields in wound healing, Curr Top Dev Biol, 58(2003) 1-26.

[11] F. Lin, F. Baldessari, C.C. Gyenge, T. Sato, R.D. Chambers, J.G. Santiago, et al., Lymphocyte electrotaxis in vitro and in vivo, The Journal of Immunology, 181(2008) 246571.

[12] D.K. Ahirwar, M.W. Nasser, T.H. Jones, E.K. Sequin, J.D. West, T.L. Henthorne, et al., Non-contact method for directing electrotaxis, Sci Rep, 5(2015).

[13] A. Jahanshahi, L.-M. Schönfeld, E. Lemmens, S. Hendrix, Y. Temel, In vitro and in vivo neuronal electrotaxis: a potential mechanism for restoration?, Mol Neurobiol, 49(2014) 100516. 
[14] X. Meng, M. Arocena, J. Penninger, F.H. Gage, M. Zhao, B. Song, PI3K mediated electrotaxis of embryonic and adult neural progenitor cells in the presence of growth factors, Exp Neurol, 227(2011) 210-7.

[15] M.K. Chaudhury, G.M. Whitesides, How to make water run uphill, Science, 256(1992) 1539-41.

[16] J.D. Smith, R. Dhiman, S. Anand, E. Reza-Garduno, R.E. Cohen, G.H. McKinley, et al., Droplet mobility on lubricant-impregnated surfaces, Soft Matter, 9(2013) 1772-80.

[17] J. Gong, C.-J.C. Kim, All-electronic droplet generation on-chip with real-time feedback control for EWOD digital microfluidics, Lab on a chip, 8(2008) 898-906.

[18] M.G. Pollack, R.B. Fair, A.D. Shenderov, Electrowetting-based actuation of liquid droplets for microfluidic applications, Appl Phys Lett, 77(2000) 1725-6.

[19] A.A. Darhuber, J.P. Valentino, S.M. Troian, S. Wagner, Thermocapillary actuation of droplets on chemically patterned surfaces by programmable microheater arrays, Journal of Microelectromechanical Systems, 12(2003) 873-9.

[20] D. Lombardi, P.S. Dittrich, Droplet microfluidics with magnetic beads: a new tool to investigate drug-protein interactions, Anal Bioanal Chem, 399(2011) 347-52.

[21] P.-Y. Chiou, Z. Chang, M.C. Wu, Droplet manipulation with light on optoelectrowetting device, Microelectromechanical Systems, Journal of, 17(2008) 133-8.

[22] A. Wixforth, C. Strobl, C. Gauer, A. Toegl, J. Scriba, Z. v Guttenberg, Acoustic manipulation of small droplets, Anal Bioanal Chem, 379(2004) 982-91.

[23] I. Lagzi, S. Soh, P. Wesson, K. Browne, B. Grzybowski, Maze solving by chemotactic droplets, J Am Chem Soc, 132(2010) 1198-9.

[24] M.G. Velarde, Drops, liquid layers and the Marangoni effect, Phil Trans R Soc A, (1998) 829-42.

[25] L. Florea, K. Wagner, P. Wagner, G.G. Wallace, F. Benito - Lopez, D.L. Officer, et al., Photo - Chemopropulsion-Light - Stimulated Movement of Microdroplets, Adv Mater, 26(2014) 7339-45.

[26] A. Diguet, R.-M. Guillermic, N. Magome, A. Saint-Jalmes, Y. Chen, K. Yoshikawa, et al., Photomanipulation of a droplet by the chromocapillary effect, Angewandte Chemie (International ed in English), 48(2009) 9281-4.

[27] M.R. Khan, C.B. Eaker, E.F. Bowden, M.D. Dickey, Giant and switchable surface activity of liquid metal via surface oxidation, Proceedings of the National Academy of Sciences, 111(2014) 14047-51.

[28] W. Francis, C. Fay, L. Florea, D. Diamond, Self-propelled chemotactic ionic liquid droplets, Chem Commun, 51(2015) 2342 - 4.

[29] K.J. Fraser, D.R. MacFarlane, Phosphonium-based ionic liquids: An overview, Aust J Chem, 62(2009) 309-21.

[30] S.P. Ong, O. Andreussi, Y. Wu, N. Marzari, G. Ceder, Electrochemical windows of room-temperature ionic liquids from molecular dynamics and density functional theory calculations, Chem Mater, 23(2011) 2979-86.

[31] J. Vaughan, D. Dreisinger, Electrodeposition of aluminum from aluminum chloridetrihexyl (tetradecyl) phosphonium chloride, J Electrochem Soc, 155(2008) D68-D72.

[32] S. Zhang, N. Sun, X. He, X. Lu, X. Zhang, Physical properties of ionic liquids: database and evaluation, J Phys Chem Ref Data, 35(2006) 1475-517.

[33] M.C. Buzzeo, C. Hardacre, R.G. Compton, Extended electrochemical windows made accessible by room temperature ionic liquid/organic solvent electrolyte systems, Chemphyschem, 7(2006) 176-80.

[34] D. Thompson, S. Coleman, D. Diamond, R. Byrne, Electronic structure calculations and physicochemical experiments quantify the competitive liquid ion association and probe 
stabilisation effects for nitrobenzospiropyran in phosphonium-based ionic liquids, PCCP, 13(2011) 6156-68.

[35] F.o. Mavré, R.K. Anand, D.R. Laws, K.-F. Chow, B.-Y. Chang, J.A. Crooks, et al., Bipolar electrodes: a useful tool for concentration, separation, and detection of analytes in microelectrochemical systems, Anal Chem, 82(2010) 8766-74.

[36] D. Morales, E. Palleau, M.D. Dickey, O.D. Velev, Electro-actuated hydrogel walkers with dual responsive legs, Soft Matter, 10(2014) 1337-48.

[37] T. Welton, Room-temperature ionic liquids. Solvents for synthesis and catalysis, Chem Rev, 99(1999) 2071-84.

[38] T. Itoh, K. Kude, S. Hayase, M. Kawatsura, Design of ionic liquids as a medium for the Grignard reaction, Tetrahedron Lett, 48(2007) 7774-7.

[39] S. Gallagher, A. Kavanagh, L. Florea, D.R. MacFarlane, K.J. Fraser, D. Diamond, Temperature and $\mathrm{pH}$ triggered release characteristics of water/fluorescein from 1-ethyl-3methylimidazolium ethylsulfate based ionogels, Chem Commun, 49(2013) 4613-5.

\section{Biographies}

Wayne Francis is a PhD student in Professor Dermot Diamond's research group, in Dublin City University. Wayne received his B.sc. (Ord) in Analytical Chemistry from Institute of Technology Tallaght and his B.sc. (Hons) in Chemical Sciences with Medicinal Chemistry from Dublin Institute of Technology. Wayne's research interests include the design, synthesis and applications of stimuli-responsive materials and his $\mathrm{PhD}$ is focused on the development of synthetic biomimetic micro "vehicles".

Dr. Klaudia Wagner received her MSc (Hons) ChE (Inorganic Chemistry) in SUoT, Gliwice, Poland and her PhD (Physical Chemistry and Technology of Polymers) in SUot, Gliwice, Poland. Klaudia's $\mathrm{PhD}$ focused on electrochemical determination of selected heavy metals and in her postdoctoral role in New Zealand she focused on electrochemical synthesis and characterisation of polythiophene derivatives. Dr. Wagner is currently a research fellow in IPRI and her research interests include Dye-sensitized solar cell: device fabrication / optimisation and conductive polymers, among others.

Dr. Stephen Beirne received his B.Eng and $\mathrm{PhD}$ from Dublin City University. Stephens PhD focused on the development of a low-power reactive wireless chemical sensing network. The system was based around commercially available wireless sensor platforms integrated with novel low-power colorimetric gas sensors. Since joining the IPRI in May 2010 he has been directly responsible for the implementation of a suite of commercial and custom additive fabrication tools. Commercial additive fabrication software and hardware have been 
supported by the Australian National Fabrication Facility and allow researchers access to a highly flexible means of device design and production.

Prof. David Officer obtained his PhD in Chemistry (1982) at Victoria Universityof Wellington, Wellington, New Zealand and was founding Director of the Nano-materials Research Centre at Massey University, Palmerston North, New Zealand(2002-2007). He is currently Professor of Organic Chemistry at the University of Wollongong and a Chief Investigator in the Australian Research Council Centre forNanostructured Electromaterials (www.electromaterials.edu.au/). He has publishedover 170 papers and 20 patents in the areas of functionalised conducting polymers,porphyrins and their application in dye sensitised solar cells, and graphene.

Prof. Gordon Wallace was awarded a PhD (1983) degree and a DSc (2000) at DeakinUniversity. He was appointed Professor at University of Wollongong in 1990. Hewas awarded an ARC Professorial Fellowship in 2002; an ARC Federation Fellowshipin 2006 and ARC Laureate Fellowship in 2011. Professor Gordon Wallace's researchinterests include advanced materials and additive fabrication and the use of these toconfront the challenges we face in energy conversion and storage as well as medicalbionic devices.

Dr. Larisa Florea studied organic chemistry and chemical engineering at University "Politehnica" from Timisoara, Romania (B.Sc. Hons 2009). In 2009 she joined the Adaptive Sensors Group at Dublin City University where she earned her Ph.D. under the supervision of Prof. Dermot Diamond and Dr. Fernando Benito-Lopez. Since 2013 she has carried out her postdoctoral research with Prof. Dermot Diamond in the INSIGHT Centre at Dublin City University, where she is currently Team Leader in smart materials and microfluidics. Her research interests include the design, synthesis and applications of stimuli-responsive polymers as novel sensing and actuating materials in microfluidic devices.

Prof. Dermot Diamond received his Ph.D. and D.Sc. from Queen's University Belfast (Chemical Sensors, 1987, Internet Scale Sensing, 2002), and was Vice-President for Research at Dublin City University (2002-2004). He has published over 300 peer-reviewed papers in international journals, is a named inventor in 18 patents, and is co-author and editor of four books. He is director and founding member of the National Centre for Sensor Research 
(www.ncsr.ie) at Dublin City University, and an SFI-funded investigator in the INSIGHT Centre (http://www.insight-centre.org). 